The Astrophysical Journal, 289:129-140, 1985 February 1

(c) 1985. The American Astronomical Society. All rights reserved. Printed in U.S.A.

\title{
THE INNER DISK OF NGC 253
}

N. Z. SCOVILLE
Palomar Observatory, California Institute of Technology; and University of Massachusetts, Amherst
B. T. SoIFER AND G. NEUGEBAUER
Palomar Observatory, California Institute of Technology
JUDITH S. YOUNG
University of Massachusetts, Amherst
K. MATTHEWS
Palomar Observatory, California Institute of Technology
AND
JAYNE YERKA
University of Massachusetts, Amherst
Received 1984 June 4 accepted 1984 August 21

ABSTRACT

The stellar and interstellar matter distributions in the luminous Sc galaxy NGC 253 are analyzed using maps of the near-infrared continuum $(\lambda=1-2 \mu \mathrm{m})$ and the millimeter $\mathrm{CO}$ emission line. The stellar disk traced out in the near-infrared exhibits two components: a nuclear peak of diameter $\sim 20^{\prime \prime}(\sim 300 \mathrm{pc})$ and an extended, inner disk of diameter $\sim 360^{\prime \prime}(\sim 6 \mathrm{kpc})$. Similar components are also evident in the molecular gas distribution.

The $2 \mu \mathrm{m}$ brightness distribution mapped over the full extent of the inner disk is dominated by a barlike feature at position angle $17^{\circ}$ east of the major axis extending to $\pm 120^{\prime \prime}$ on opposite sides of the nucleus. The $\mathrm{CO}$ distribution exhibits a weak secondary peak just outside the bar.

The derived abundance of molecular gas in the inner disk exceeds that of $\mathrm{H}$ I by a factor of 10 and exhibits a much steeper falloff with radius. The total mass of $\mathrm{H}_{2}$ inside $R=4 \mathrm{kpc}$ is $\sim 2 \times 10^{9} M_{\odot}$, which is $\sim 7 \%$ of the dynamical mass at the same radius. If the observed far-infrared luminosity in the central $R \leq 500 \mathrm{pc}$ is produced by young stars, the minimum rate of OBA star formation is $2.3 M_{\odot} \mathrm{yr}^{-1}$, and the observed molecular clouds will be cycled into stars in $\lesssim 3 \times 10^{8} \mathrm{yr}$. It is speculated that the bar, detected in the near-infrared map, could feed fresh molecular gas from the outer disk to the active, star-forming nucleus.

Subject headings: galaxies: individual — galaxies: nuclei — galaxies: stellar content — infrared: sources — interstellar: matter — interstellar: molecules — stars: formation

\section{INTRODUCTION}

A major enigma in our understanding of galaxies is the energetic activity discovered in their nuclei. In many instances, extraordinarily high luminosities of $10^{9}-10^{11} L_{\odot}$ are emitted at far-infrared wavelengths within the central few kiloparsecs. The correlation of this luminosity with the presence of abundant dust clouds and optical $H$ II regions suggests that the source of energy may often be a young stellar generation with a high proportion of massive stars (e.g., Rieke et al. 1980). Whether the high rate of star formation is due to an especially abundant interstellar medium in these galaxies or whether the given supply is used with a greater efficiency is presently unknown. In addition, the more fundamental question of how the high level of nuclear activity is linked to the structure of the outer galactic disk and the galactic environment remains virtually unexplored.

A particularly clear example of these phenomena is offered by the southern Sc spiral galaxy NGC 253 . In the nucleus of this galaxy $(R<500 \mathrm{pc})$, the far-infrared luminosity is $\sim 3 \times 10^{10} L_{\odot}$ (Telesco and Harper 1980), and noncircular motions of approximately $100 \mathrm{~km} \mathrm{~s}^{-1}$ are observed in optical emission lines (Ulrich 1978). NGC 253 also shows extremely bright optical and infrared emission lines (Demoulin and Burbidge 1970; Wynn-Williams et al. 1979; Beck, Lacy, and
Geballe 1979), requiring an ionizing flux in the central $150 \mathrm{pc}$ equivalent to $1000 \mathrm{O6}$ stars (Ulrich 1978; Turner and Ho 1983). It is also exceptionally rich in molecular gas - the CO emission line is just as bright as in M82 (Rickard et al. 1977). Recent X-ray data have revealed a complex of point sources and diffuse emission in the central $2 \mathrm{kpc}$ of NGC 253 (Fabbiano and Trinchieri 1984). Surprisingly, the disk of this galaxy, outside $2 \mathrm{kpc}$ radius, shows no evidence of peculiar activity or disruption.

In this article we present maps of the near-infrared continuum and millimeter $\mathrm{CO}$ line emission from the inner disk of NGC 253. The infrared maps serve to define the structure of the stellar disk, relatively free of obscuration by dust clouds; the CO data serve to trace out the radial distribution of starforming material, that is, the molecular hydrogen clouds.

\section{OBSERVATIONS}

\section{a) Near-Infrared Photometric Mapping}

Near-infrared $(\lambda=1-2 \mu \mathrm{m})$ photometry and maps were obtained in 1982 using the $5 \mathrm{~m}$ Hale telescope at Palomar. All measurements were made using a single-element detector located behind a $J(1.27 \mu \mathrm{m}, \Delta \lambda \doteq 0.24 \mu \mathrm{m}), H(1.65 \mu \mathrm{m}$, $\Delta \lambda=0.32 \mu \mathrm{m})$, or $K(2.2 \mu \mathrm{m}, \Delta \lambda=0.40 \mu \mathrm{m})$ photometric filter. 
TABLE 1

AdOPTED Parameters FOR NGC 253

\begin{tabular}{|c|c|}
\hline Parameter & Value \\
\hline R.A. $(1950)^{a}$ & $00^{\mathrm{h}} 45^{\mathrm{m}} 05^{\mathrm{s}} 8$ \\
\hline Decl. $(1950)^{\mathrm{a}} \ldots \ldots \ldots \ldots \ldots$ & $-25^{\circ} 33^{\prime} 38^{\prime \prime}$ \\
\hline Systemic velocity (LSR) ${ }^{\mathrm{b}}$ & $229 \mathrm{~km} \mathrm{~s}^{-1}$ \\
\hline Distance $^{c} \ldots \ldots \ldots \ldots \ldots$ & $3.4 \mathrm{Mpc}$ \\
\hline Inclination $^{\mathrm{d}} \ldots \ldots \ldots \ldots \ldots \ldots \ldots \ldots$ & 78.5 \\
\hline Position angle of major axis ${ }^{d} \ldots \ldots \ldots \ldots \ldots \ldots$ & $51^{\circ}$ \\
\hline \multicolumn{2}{|l|}{ Beam size on galaxy $\ldots \ldots \ldots \ldots \ldots \ldots \ldots \ldots \ldots \ldots$} \\
\hline $10^{\prime \prime}($ near-infrared $) \ldots \ldots \ldots \ldots \ldots \ldots \ldots \ldots$ & $165 \mathrm{pc}$ \\
\hline 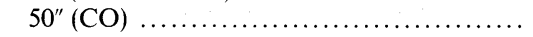 & $825 \mathrm{pc}$ \\
\hline
\end{tabular}

a Becklin, Fomalont, and Neugebauer 1973.

${ }^{b}$ Corresponds to $V_{\odot}=236 \mathrm{~km} \mathrm{~s}^{-1}$ (Pence 1981).

c Sandage and Tammann 1975

d Pence 1981.

The photometry is based on comparison with stars listed by Elias et al. (1982). Chopping was done at $5 \mathrm{~Hz}$ using the $\mathrm{f} / 70$ infrared secondary, chopping parallel to the minor axis to a reference position 300" away. In all cases there was negligible contamination due to flux from the region between the signal and reference positions. All observations were obtained with a $10^{\prime \prime}$ focal-plane aperture, corresponding to a linear size of 165 pc at the distance of $3.4 \mathrm{Mpc}$ (see Table 1 for adopted parameters of NGC 253). Photometric flux measurements obtained on the nucleus and at offsets of $30^{\prime \prime}$ on the major and minor axes are listed in Table 2.

Scans at $J, H$, and $K$ were taken along the major axis to a distance of $\pm 500^{\prime \prime}$ from the nucleus and along the minor axis to a distance of $\pm 150^{\prime \prime}$ from the nucleus. At the ends of the scans the signal was in all cases less than $1 \%$ of the peak on the nucleus. In addition to the principal axis scans, the central disk of NGC 253 was mapped at $2.2 \mu \mathrm{m}$ out to a distance of $150^{\prime \prime}$ along the major axis. A map was constructed from 31 scans made parallel to the minor axis, extending to $\pm 100^{\prime \prime}$ from the major axis. All scans were made by moving the telescope at a

TABLE 2

INFRARED FLUXES

\begin{tabular}{cccc}
\hline \hline Position & $\begin{array}{c}\text { Wavelength } \\
(\mu \mathrm{m})\end{array}$ & Magnitude & $\begin{array}{c}\text { Flux } \\
\text { Density } \\
(\mathrm{mJy})\end{array}$ \\
\hline Nucleus $\ldots \ldots \ldots \ldots \ldots$ & 1.27 & $9.64 \pm 0.09$ & 220 \\
& 1.65 & $8.57 \pm 0.08$ & 391 \\
& 2.2 & $7.77 \pm 0.07$ & 504
\end{tabular}

$\begin{array}{llll}\text { Major axis: } & & & \\ 30^{\prime \prime} \mathrm{NE} \ldots \ldots \ldots \ldots & 1.27 & 11.58 \pm 0.09 & 37 \\ & 1.65 & 10.83 \pm 0.08 & 49 \\ 30^{\prime \prime} \mathrm{SW} \ldots \ldots \ldots \ldots & 2.2 & 10.42 \pm 0.07 & 44 \\ & 1.27 & 11.94 \pm 0.09 & 26 \\ & 1.65 & 11.04 \pm 0.08 & 40 \\ & 2.2 & 10.49 \pm 0.07 & 41\end{array}$

$\begin{array}{llll}\text { Minor axis: } & & & \\ 30^{\prime \prime} \mathrm{NW} \ldots \ldots \ldots \ldots & 1.27 & 12.26 \pm 0.09 & 20 \\ & 1.65 & 11.49 \pm 0.08 & 27 \\ 30^{\prime \prime} \mathrm{SE} \ldots \ldots \ldots \ldots & 2.2 & 11.05 \pm 0.07 & 25 \\ & 1.27 & 12.15 \pm 0.09 & 22 \\ & 1.65 & 11.47 \pm 0.08 & 27 \\ & 2.2 & 11.15 \pm 0.07 & 22\end{array}$

${ }^{\text {a }}$ Flux densities at $0.0 \mathrm{mag}$ are assumed to be $1578 \mathrm{Jy}, 1041 \mathrm{Jy}$, and $646 \mathrm{Jy}$ at $1.27 \mu \mathrm{m}, 1.65 \mu \mathrm{m}$, and $2.2 \mu \mathrm{m}$. rate of $1^{\prime \prime} \mathrm{s}^{-1}$ relative to the galaxy and sampling the signal every second.

\section{b) 2.6 Millimeter $\mathrm{CO}$ Observations}

Observations of the $2.6 \mathrm{~mm} \mathrm{CO}(J=1 \rightarrow 0)$ emission were obtained in 1982 using the $14 \mathrm{~m}$ telescope of the Five College Radio Astronomy Observatory (FCRAO). ${ }^{1}$ A cooled mixer $\left(T_{\mathrm{SSB}}=500 \mathrm{~K}\right)$ was used at the Cassegrain focus with spectral resolution provided by a $256 \times 1 \mathrm{MHz}$ channel filter bank (1 $\mathrm{MHz}$ corresponds to $\Delta V=2.6 \mathrm{~km} \mathrm{~s}^{-1}$ at the $\mathrm{CO}$ frequency, $115.2712 \mathrm{GHz}$ ). The half-power beamwidth of the telescope is $50^{\prime \prime}$, or $825 \mathrm{pc}$ in NGC 253. Pointing errors are typically $5^{\prime \prime} \mathrm{rms}$. The intensity calibration procedure is described in detail in Young and Scoville (1982). On this scale the center of the Orion Nebula $(\mathrm{BN}-\mathrm{KL})$ has $T_{A}^{*}(\mathrm{CO})=62 \mathrm{~K}$.

The observations along the major axis extend out to a distance of $315^{\prime \prime}$ from the nucleus; additional spectra were obtained on the minor axis and along the diagonals at angles of $45^{\circ}$ with respect to the major axis. Within the central $3^{\prime}$ the data are spaced by $22^{\prime \prime}$, while farther out they are spaced by $45^{\prime \prime}$.

Table 3 lists the measured line parameters as a function of offset $(\Delta \alpha, \Delta \delta)$ from the $\lambda=2.2 \mu \mathrm{m}$ peak (Becklin, Fomalont, and Neugebauer 1973). The line parameters include the peak antenna temperature $\left(T_{A}^{*}\right)$, integrated line flux $\left(I_{\mathrm{Co}} \equiv \int T_{A}^{*} d V\right)$, peak velocities, mean velocities, and line widths (FWHM) for each profile. The 2 sigma limit on the line integrals amounts to $2.5 \mathrm{~K} \mathrm{~km} \mathrm{~s}^{-1}$; at all but three of the 33 observed positions, significant $\mathrm{CO}$ emission was detected.

\section{OBSERVATIONAL RESULTS}

\section{a) Intensity Profiles on Major Axis}

The near-infrared strip scans at $\lambda=1.27$ and $2.2 \mu \mathrm{m}$ along the major axis are shown at the same angular scale as the optical photograph in Figure 1. A contour map of the $2.6 \mathrm{~mm}$ $\mathrm{CO}$ emission velocities along the major axis is also provided in Figure 1. Both the near-infrared and $\mathrm{CO}$ emission are strongly peaked at the center of the galaxy with a symmetric falloff on each side. The infrared scans in Figure 1 clearly show the prescence of two distinct components: a bright nuclear source of width $\sim 20^{\prime \prime}$ and a plateau, or inner disk, of width $\sim 360^{\prime \prime}$. These components may also be seen in the $\mathrm{CO}$ data but with poorer definition presumably because of the coarser angular resolution.

The similarity between the infrared light profile and the $\mathrm{CO}$ is especially evident when the $2.2 \mu \mathrm{m}$ data are smoothed to a resolution comparable with the $50^{\prime \prime} \mathrm{CO}$ beam. In Figure 2 both the integrated $\mathrm{CO}$ emission, $I_{\mathrm{CO}}$, and the $2.2 \mu \mathrm{m}$ radiation are shown with the $2.2 \mu \mathrm{m}$ data filtered to $50^{\prime \prime}$ resolution along the scan (still $10^{\prime \prime}$ resolution in the orthogonal direction). Both the $\mathrm{CO}$ and the smoothed $2.2 \mu \mathrm{m}$ data show nuclear and disk components with similar relative amplitude. In addition, the $\mathrm{CO}$ shows a secondary maximum at approximately $200^{\prime \prime}$ radius, perhaps slightly outside the edge of the infrared plateau. Along the minor axis the $\mathrm{CO}$ resolution is too low to provide a meaningful comparison with the infrared light profiles shown in Figure 3.

The existence of distinct nuclear and disk components in NGC 253 is also evident in the $J-H$ and $H-K$ colors shown

\footnotetext{
${ }^{1}$ The FCRAO is operated with support from the National Science Foundation under grant AST825702 and with permission of the Metropolitan District Commission, Commonwealth of Massachusetts.
} 
TABLE 3

NGC 253 CO Line Parameters

\begin{tabular}{|c|c|c|c|c|c|}
\hline Position & $\begin{array}{l}T_{A}^{*} \\
(\mathrm{~K})\end{array}$ & $\begin{array}{c}I_{\mathrm{CO}^{\mathrm{a}}} \\
\left(\mathrm{K} \mathrm{km} \mathrm{s}^{-1}\right)\end{array}$ & $\begin{array}{c}V_{\text {peak }}^{\mathrm{b}} \\
\left(\mathrm{km} \mathrm{s}^{-1}\right)\end{array}$ & $\begin{array}{c}V_{\text {mean }}^{\mathrm{b}} \\
\left(\mathrm{km} \mathrm{s}^{-1}\right)\end{array}$ & $\begin{array}{c}\Delta V^{\mathrm{b}} \\
\left(\mathrm{km} \mathrm{s}^{-1}\right)\end{array}$ \\
\hline \multicolumn{6}{|c|}{ Major $\mathrm{Axis}^{\mathrm{c}}$} \\
\hline$+315^{\prime \prime}$. & 0.11 & 7 & 50 & 45 & 62 \\
\hline$+270^{\prime \prime}$. & $<0.1$ & $<3$ & & & \\
\hline $225^{\prime \prime}$ & 0.30 & 18 & 50 & 82 & 60 \\
\hline $180^{\prime \prime}$. & 0.47 & 26 & 50 & 74 & 55 \\
\hline $135^{\prime \prime}$. & 0.32 & 17 & 70 & 83 & 52 \\
\hline $90^{\prime \prime}$. & 0.23 & 27 & 210 & 159 & 116 \\
\hline $68^{\prime \prime}$. & 0.25 & 40 & 150 & 155 & 166 \\
\hline $45^{\prime \prime}$. & 0.42 & 77 & 160 & 163 & 184 \\
\hline $22^{\prime \prime}$. & 0.88 & 168 & 170 & 210 & 191 \\
\hline ....... & 1.25 & 272 & 290 & 261 & 217 \\
\hline$-22^{\prime \prime}$. & 0.79 & 126 & 300 & 292 & 159 \\
\hline$-45^{\prime \prime}$. & 0.41 & 54 & 300 & 309 & 129 \\
\hline$-68^{\prime \prime}$ & 0.28 & 25 & 310 & 304 & 88 \\
\hline$-90^{\prime \prime}$ & 0.17 & 13 & 440 & 400 & 75 \\
\hline$-135^{\prime \prime}$. & 0.36 & 10 & 420 & 400 & 28 \\
\hline$-180^{\prime \prime}$. & 0.45 & 15 & 430 & 417 & 34 \\
\hline$-225^{\prime \prime}$. & 0.19 & 18 & 420 & 401 & 93 \\
\hline$-270^{\prime \prime} \ldots \ldots \ldots \ldots$ & 0.12 & 6 & 410 & 411 & 50 \\
\hline$-315^{\prime \prime} \ldots \ldots \ldots \ldots$ & $<0.10$ & $<3$ & $\ldots$ & $\ldots$ & $\ldots$ \\
\hline
\end{tabular}

\begin{tabular}{|c|c|c|c|c|c|}
\hline \multicolumn{6}{|c|}{ Minor Axis ${ }^{c}$} \\
\hline $90^{\prime \prime} \ldots \ldots \ldots \ldots$ & $<0.1$ & $<3$ & & & \\
\hline $45^{\prime \prime}$ & 0.38 & 47 & 190 & 233 & 124 \\
\hline $22^{\prime \prime}$. & 1.00 & 195 & 300 & 274 & 195 \\
\hline 0 & 1.25 & 272 & 290 & 261 & 217 \\
\hline$-22^{\prime \prime}$ & 0.62 & 113 & 300 & 261 & 183 \\
\hline$-45^{\prime \prime}$ & 0.22 & 22 & 250 & 270 & 99 \\
\hline$-90^{\prime \prime} .$. & 0.29 & 19 & 230 & 261 & 66 \\
\hline \multicolumn{6}{|c|}{ SW-NE Diagonal $^{c}$} \\
\hline $45^{\prime \prime}$ & 0.48 & 81 & 150 & 226 & 167 \\
\hline $22^{\prime \prime}$ & 1.01 & 215 & 280 & 233 & 215 \\
\hline ...... & 1.25 & 272 & 290 & 261 & 217 \\
\hline$-22^{\prime \prime}$ & 0.69 & 111 & 310 & 289 & 162 \\
\hline$-45^{\prime \prime} .$. & 0.41 & 68 & 360 & 322 & 165 \\
\hline \multicolumn{6}{|c|}{ SE-NW Diagonal $^{\mathfrak{c}}$} \\
\hline$+45^{\prime \prime}$ & 0.38 & 52 & 260 & 312 & 136 \\
\hline$+22^{\prime \prime}$ & 1.15 & 210 & 310 & 295 & 182 \\
\hline $0 \ldots \ldots \ldots$ & 1.25 & 272 & 290 & 261 & 217 \\
\hline$-22^{\prime \prime} \ldots \ldots \ldots \ldots$ & 0.60 & 101 & 250 & 248 & 167 \\
\hline$-45^{\prime \prime} \ldots \ldots \ldots \ldots$ & 0.45 & 63 & 270 & 220 & 141 \\
\hline
\end{tabular}

${ }^{a}$ Upper limits are $2 \sigma$.

b Velocities are with respect to the local standard of rest (LSR).

${ }^{c}$ Major axis at P.A. $=51^{\circ} ;$ minor axis at P.A. $=-39^{\circ} ;$ SW-NE diagonal at P.A. $=6^{\circ} ;$ and SE-NW diagonal at P.A. $=-84^{\circ}$. Positive offset is always on north side of the center along each track.

in Figure 4. The nucleus is seen to be extremely red compared with the disk. On the center position, $J-H=1.1 \mathrm{mag}$, which is 0.4 mag redder than the disk light. The spatial extent of this red nuclear component is not symmetric on the nucleus. The excessively red colors may be seen out to approximately $50^{\prime \prime}$ on the southwest but to just $20^{\prime \prime}$ on the northeast side of the nucleus. This asymmetry is similar to that seen in the spatial distribution of radio $\mathrm{H}$ II regions by Turner and Ho (1983). Throughout the remainder of the disk, the colors are fairly constant: $\langle J-H\rangle=0.7 \mathrm{mag}$ and $\langle H-K\rangle=0.4 \mathrm{mag}$. The only notable departures occur at $-100^{\prime \prime} \mathrm{SW}$ and $+120^{\prime \prime} \mathrm{NE}$ of the nucleus; the latter coincides with a star on the edge of the scan path, and thus the color there is susceptible to small tracking errors.

Figure 5 shows a $J-H$ versus $H-K$ plot for the data on the major axis. For comparison we have also included in this plot the large-aperture photometry of Aaronson (1977) transformed to the CIT photometric system. To make a direct comparison between the observations, we have synthesized from our data an aperture equal to the $105^{\prime \prime}$ aperture of Aaronson. This was done by averaging the scans along the major and minor axes to derive a mean flux density versus radius, and then integrating this to a radius corresponding to the Aaronson aperture to derive a flux in the corresponding circular aperture at $J, H$, and $K$. The large-aperture colors derived from the major and minor axes scans in this way are also plotted in Figure 5 and show excellent agreement with the photometric measurements of Aaronson. This comparison indicates that accurate colors can be derived from the scans of the galaxy, but it also underscores the problem that a large-aperture, averaging over a range of conditions, may not properly represent the local colors. The 1 sigma noise on the observed colors is $\lesssim 0.03 \mathrm{mag}$ out to offsets of $200^{\prime \prime}$. Also shown are the giant and dwarf sequences for stellar spectral classes K0 to M8 (Frogel et al. 1978) and the reddening vector corresponding to $A_{V}=1$ mag taken from Cohen et al. (1981).

The cross indicates the mean colors for late-type spiral galaxies $(J-H=0.67 \mathrm{mag}, H-K=0.23 \mathrm{mag}$; Aaronson 1978 transformed into the CIT photometric system); the mean colors for other spiral types differ by less than 0.03 mag in $J-H$ and $H-K$ from those shown. In the nucleus and throughout much of the inner disk in $H-K$ color is considerably redder (by $0.2 \mathrm{mag}$ ) than a normal stellar disk, or even a disk enriched in late-type giant stars and suffering foreground dust extinction. This fact is clear since the reddening vector translates points to the upper right in Figure 5 but not enough to the right. On the nucleus, the observed colors $H-K$ and $J-H$ are redder by 0.5 and $0.4 \mathrm{mag}$, respectively, than the normal Sc galaxy colors. The enrichment of the Sc population with more late-type supergiant stars as suggested by Rieke et al. (1980) to model the near-infrared spectral features cannot alleviate the problem since the colors of the supergiants are, in fact, similar to the Sc colors.

To account for the nuclear colors requires the addition of a component such as warm dust or free-free radiation, which contributes much more at $2.2 \mu \mathrm{m}$ than at the shorter wavelengths. The free-free component has been estimated by WynnWilliams et al. (1979) to be $\sim 50 \mathrm{mJy}$ at $\lambda=2.2 \mu \mathrm{m}$ in a $10^{\prime \prime}$ diameter diaphragm based on the Bry line flux. A similar value is obtained from Turner and Ho's (1983) free-free estimate at $\lambda=2 \mathrm{~cm}$. After correction for extinction at $2 \mu \mathrm{m}$, we conclude that the free-free contribution must be an insignificant part, $\sim 25 \mathrm{mJy}$, of the total $504 \mathrm{mJy}$ observed.

On the other hand, hot dust, presumably located in the nuclear $\mathrm{H}$ II regions, is a viable source of the $2 \mu \mathrm{m}$ excess. Hot dust at $600-1000 \mathrm{~K}$ will translate the Sc galaxy point almost directly to the right in the color-color plot (see Aaronson 1978). The combination of approximately $4 \mathrm{mag}$ of visual extinction and $200 \mathrm{mJy}$ of dust emission at $\lambda=2 \mu \mathrm{m}$ on top of the standard Sc color would account for the observed nuclear colors. This is in agreement with the measurements of Becklin, Fomalont, and Neugebauer (1973). Their 3.5 and $10.1 \mu \mathrm{m}$ fluxes give a color temperature of $\sim 500 \mathrm{~K}$ for circular regions centered on the nucleus of radii from $5^{\prime \prime}$ to $30^{\prime \prime}$ diameter. Since the hot dust inferred from this temperature will not be isothermal, its hotter components near $1000 \mathrm{~K}$ will produce a $2.2 \mu \mathrm{m}$ excess. In fact, our derived $2.2 \mu \mathrm{m}$ excess, combined with their $3.5 \mu \mathrm{m}$ flux, has a color temperature slightly below $1000 \mathrm{~K}$. 

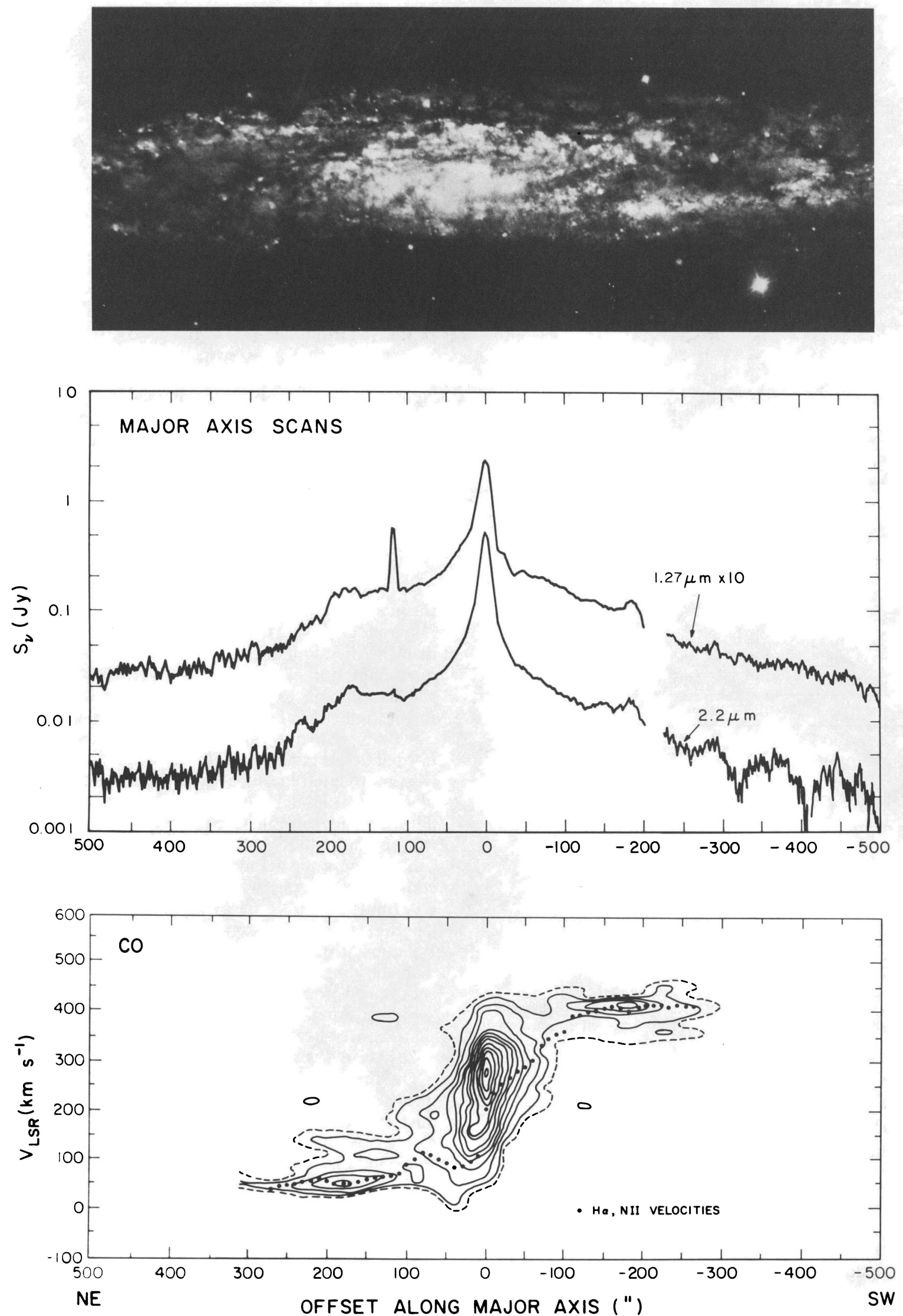

FIG. 1.-(top) Enlargement of NGC 253 photograph (reprinted, by permission, from Sandage 1961). (middle) The $\lambda=1.27$ and $2.2 \mu \mathrm{m}$ flux density in $10^{\prime \prime}$ diameter diaphragm is plotted logarithmically for the major axis scans in NGC 253. (bottom) The spatial-velocity map of $2.6 \mathrm{~mm}$ CO emission is shown for the major axis of NGC 253. Superposed are the optical velocities ( $\mathrm{H} \alpha$ and N II), measured by Ulrich (1978) and Pence (1981). Contour levels are at $\Delta T_{A}^{*}=0.1 \mathrm{~K}$, and the outer dashed contour is at $0.05 \mathrm{~K}$. 


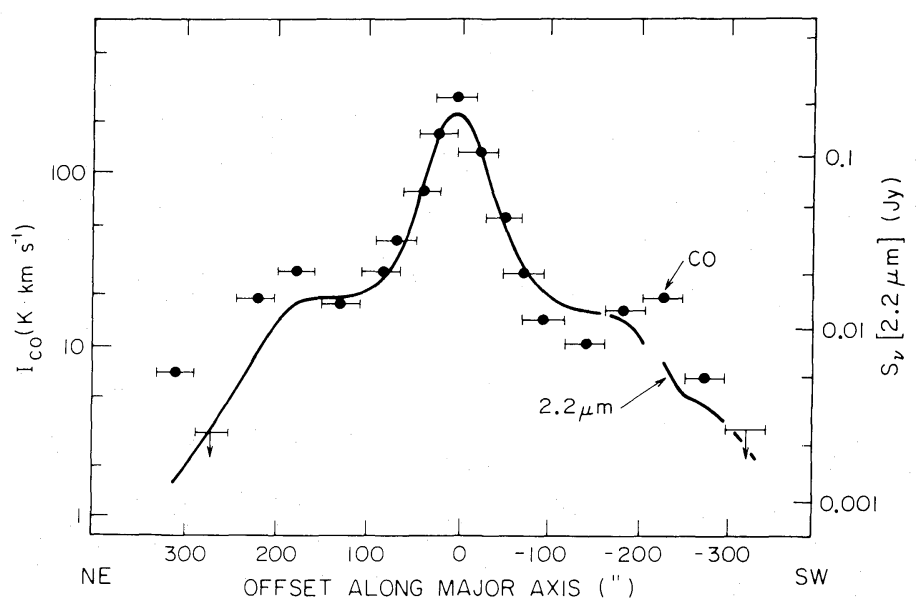

FIG. 2.-The integrated $\mathrm{CO}$ line flux, $I_{\mathrm{CO}}$, is compared with the $\lambda=2.2 \mu \mathrm{m}$ flux density along the major axis. The $\mathrm{CO}$ measurements are at $50^{\prime \prime}$ resolution; the 2.2 $\mu \mathrm{m}$ data, obtained with a $10^{\prime \prime}$ diameter diaphragm, were filtered to a $50^{\prime \prime}$ resolution along the scan line, giving an effective beam $50^{\prime \prime} \times 10^{\prime \prime}$ though the flux density units are per $10^{\prime \prime}$ beam area.

Since the disk colors, observed off the nucleus, are less extreme, these points can be matched with smaller amounts of extinction and hot dust contributions. It is noteworthy that the points immediately SW of the nucleus $\left(-10^{\prime \prime},-20^{\prime \prime},-30^{\prime \prime}\right.$, and $-40^{\prime \prime}$ ), which are to the upper right in Figure 5, suggestive of large extinction, also coincide with a dark dust lane in Figure 1. It is also interesting that the points outside the nucleus with greatest $2.2 \mu \mathrm{m}$ excess (e.g., -150 to $-190^{\prime \prime}$ ) coincide with bright optical emission regions ( $\mathrm{H}$ II) seen in Figure $1 \mathrm{SW}$ of the nucleus where there should be large quantities of hot dust. In this region the fractional $2.2 \mu \mathrm{m}$ excess due to hot dust must be about the same as in the nuclear region.

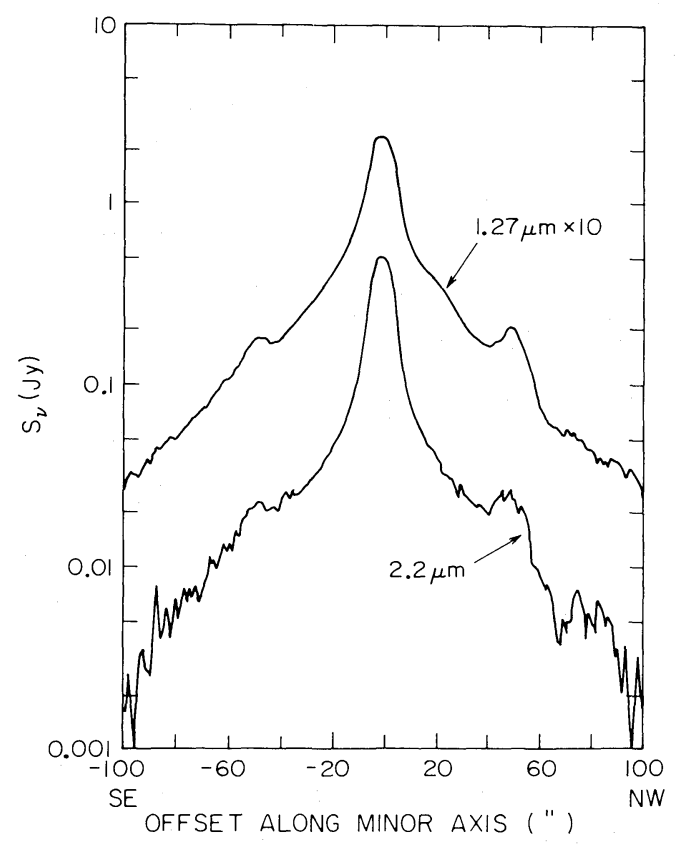

FIG. 3

\section{b) Map of the Inner Disk}

To define the structure of the inner disk, a completely sampled map was obtained at $2.2 \mu \mathrm{m}$ with $10^{\prime \prime}$ resolution; Figure 6 shows these data with contours spaced every $0.25 \mathrm{mag}$ superposed on the optical image of the galaxy. The principal features of this map are the nuclear source, of half-power width $10^{\prime \prime} \times 20^{\prime \prime}$, and the extended disk, within which one clearly sees a linear, barlike feature. The observed position angle, $17^{\circ}$ east of the major axis, implies that the bar makes an angle of $33^{\circ}$

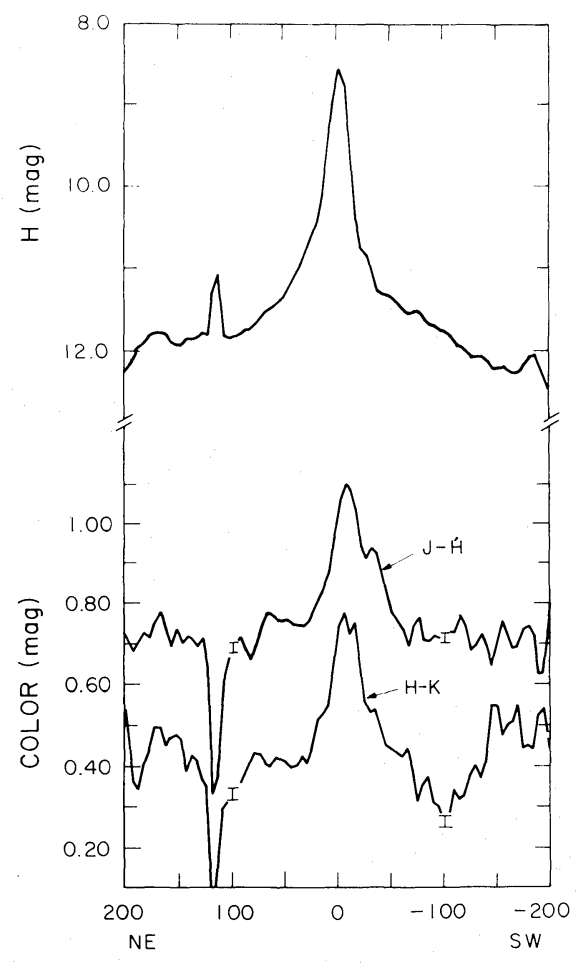

OFFSET ALONG MAJOR AXIS (")

FIG. 4

FIG. 3.-The $\lambda=1.27$ and $2.2 \mu \mathrm{m}$ flux densities along the minor axis in a $10^{\prime \prime}$ diameter diaphragm are plotted logarithmically.

Fig. 4. $-J-H$ and $H-K$ colors for the major axis of NGC 253. The dip occurring at offset $+120^{\prime \prime}$ is due to a star on the edge of the scan line; thus the colors are not reliable there. 


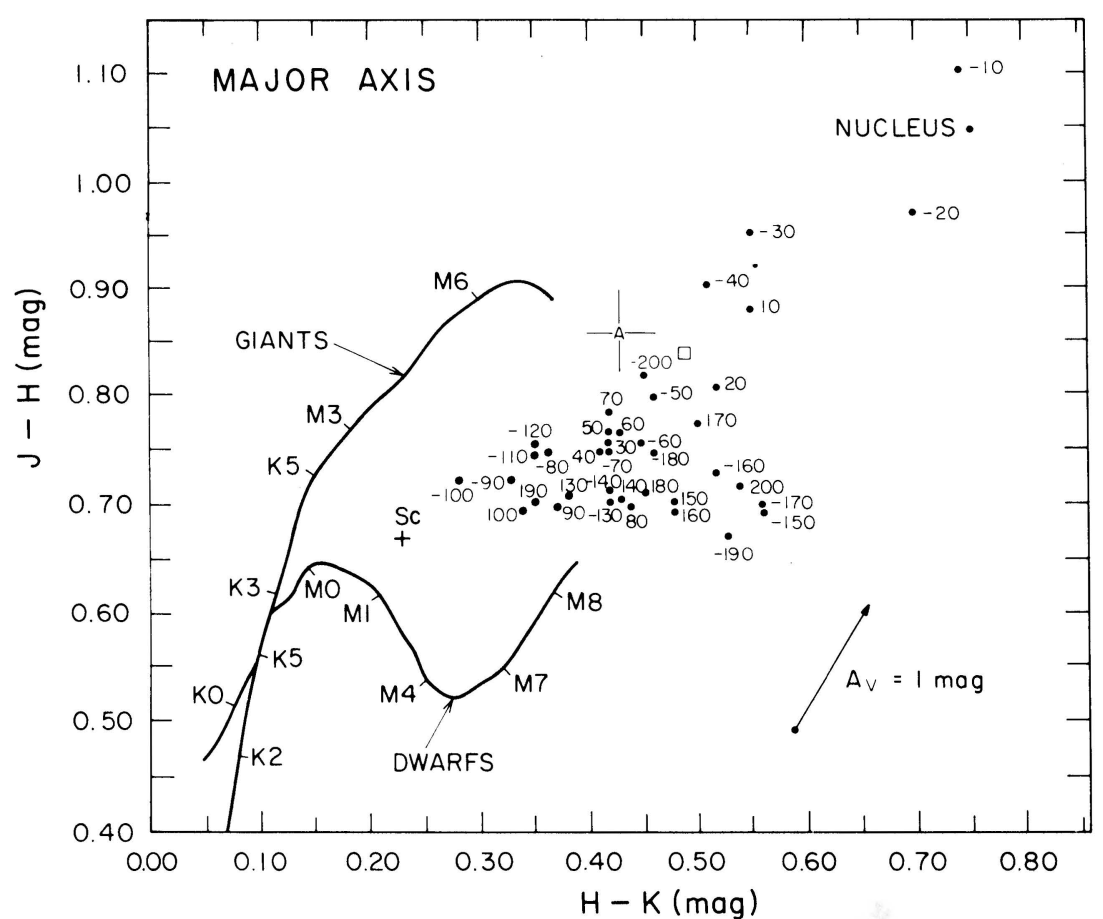

Fig. 5.-Color-color plot for major axis data (filled circles) shown in Fig. 4. Also shown are the mean color sequences for giant and dwarf stars (lines) and for a normal Sc galaxy (plus) and 105" aperture photometry on the nucleus of NGC 253 (A) (Aaronson 1977). The colors from Aaronson have been corrected to correspond to the CIT magnitude system (Elias et al. 1982). The square represents the synthetic 105" aperture data from the major and minor axes scans for comparison to the photometry of Aaronson. (See text.) The reddening vector corresponding to $A_{v}=1 \mathrm{mag}$ is from Cohen $e$ t al. (1981).

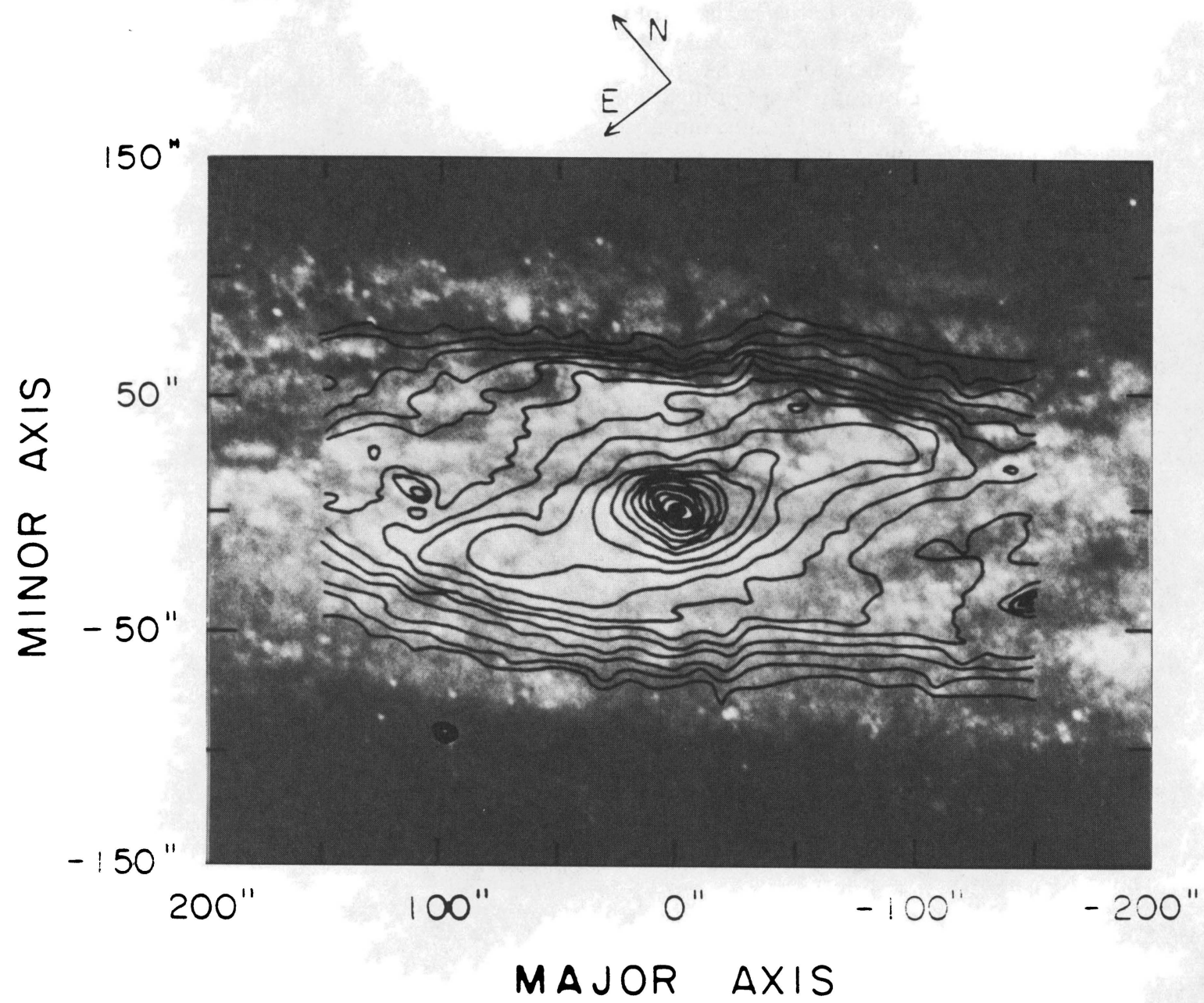

Fig. 6.-Map at $\lambda=2.2 \mu \mathrm{m}$ of the inner disk of NGC 253 at $10^{\prime \prime}$ resolution. Note the bar appearing in the infrared at position angle $17^{\circ}$ east of the major axis. Contours are logarithmically spaced by $0.25 \mathrm{mag}$. 
with respect to the minor axis in the plane of the galaxy. The extent of the bar, approximately $240^{\prime \prime}$ on the sky, corresponds to a radius of $3.5 \mathrm{kpc}$ in the disk of the galaxy.

The existence of a bar in NGC 253 has been suggested earlier by Pence (1980), who deprojected an optical photograph to reveal an elongation at position angle $\sim 80^{\circ}$, that is, an angle $29^{\circ}$ east of the major axis. Pence's (1981) kinematic study also suggested a bar since streaming motions similar to those seen in barred galaxies were observed. The fact that the 2 $\mu \mathrm{m}$ map shows this bar more clearly than the optical photographs can be attributed to the greatly reduced extinction at $2 \mu \mathrm{m}$, implying that the $2 \mu \mathrm{m}$ flux is a much better probe of the underlying disk morphology.

\section{c) $\mathrm{CO}$ Kinematics}

The $\mathrm{CO}$ velocities shown in Figure 1 may well provide a more reliable probe of the disk kinematics than the optical emission lines in view of the heavy, uneven obscuration in NGC 253. The maximum velocity spread in $\mathrm{CO}$ is seen at the center position, where $\Delta V=217 \mathrm{~km} \mathrm{~s}^{-1}$; outside $100^{\prime \prime}$ radius the line widths are generally less than $50 \mathrm{~km} \mathrm{~s}^{-1}$ (see Table 3). Across the nucleus a steep gradient in radial velocity is produced by the galactic rotation, and probably much of the large dispersion observed at the center can be attributed to the $\mathrm{CO}$ beam smearing the rotational kinematics. Within $150^{\prime \prime}$ of the center, the $\mathrm{CO}$ data indicate asymptotic velocities of $50 \mathrm{~km} \mathrm{~s}^{-1}$ (on the northeast side of the nucleus; see Fig. 1) and $420 \mathrm{~km}$ $\mathrm{s}^{-1}$ (on the southwest side).

The optical $\mathrm{H} \alpha$ and $\mathrm{N}$ II velocities measured by Ulrich (1978) and Pence (1981) along the major axis are also shown in Figure 1. Comparing the $\mathrm{CO}$ and optical velocities, one sees good agreement except at the points $-60^{\prime \prime}$ (southwest) and $+90^{\prime \prime}$ (northeast) of the nucleus. Since these positions coincide with strong optical obscuration, the $\mathrm{CO}$ probably provides a better indication of the true rotational velocities in the disk. This is also suggested by the greater axisymmetry seen in the $\mathrm{CO}$ velocities.

In Figure 7, the distribution of total $\mathrm{CO}$ line flux and the $\mathrm{CO}$ mean velocities are superposed on the optical photograph and the isovelocity contours measured by Pence (1981). Comparing the $\mathrm{CO}$ and optical velocities off the major axis, it is seen that in the southeast there is good agreement between the two, but to the northwest, discrepancies of the order of $70 \mathrm{~km} \mathrm{~s}^{-1}$ occur. The explanation for these differences surely is the presence of heavy obscuration, which is much greater on the northwest side of the minor axis than elsewhere. It is interesting that this asymmetry is also evident in the abundance of molecular gas. The integrated intensities (Fig. $7 b$ ) tend to be higher by a factor of 2 to the northwest of the nucleus compared with the southeast. This is true of all three of the inner points on each side of the nucleus. The correlation of the $\mathrm{CO}$ line flux with the strong obscuration on the northwest suggests that the $\mathrm{CO}$ emission is in fact arising from this dust lane. The kink observed in the optical isovelocity contour at $230 \mathrm{~km} \mathrm{~s}^{-1}$ on the northwest side of the nucelus is therefore probably a bias due to foreground extinction and may not represent the galactic rotation along the major axis.

In view of the large rotational gradient and possible noncircular motions, the $\mathrm{CO}$ line measured in a large beam on the nucleus does not provide a reliable indication of the galactic systemic velocity. Fortunately, two alternative approaches provide nearly identical results. On the one hand, a simple average of the asymptotic velocities obtained from the rotation curve symmetrically on opposite sides of the nucleus may be used. Adopting this approach, Pence (1981) deduced a systemic heliocentric velocity of $235 \mathrm{~km} \mathrm{~s}^{-1}\left(V_{\mathrm{LSR}}=229 \mathrm{~km} \mathrm{~s}^{-1}\right)$. A similar procedure applied to the $\mathrm{CO}$ measurements between $180^{\prime \prime}$ and $225^{\prime \prime}$ from the nucleus yields $V_{\mathrm{LSR}}=238 \mathrm{~km} \mathrm{~s}^{-1}$ (based on the peak velocities) or $244 \mathrm{~km} \mathrm{~s}^{-1}$ (based on the mean velocities). Alternatively, one may estimate the systemic velocity from near-infrared spectroscopy obtained on the nucleus. Averaging measurements of the $12.8 \mu \mathrm{m}$ Ne II emission from the central 25", Beck, Lacy, and Geballe (1979) estimated a systemic velocity of $V_{\mathrm{LSR}}=245 \mathrm{~km} \mathrm{~s}^{-1}$. Recently Kleinmann, Hall, and Scoville (1984) have measured the $\mathrm{Br} \gamma$ line at $\lambda=2.06 \mu \mathrm{m}$ in a 3.75 diameter region centered on the nucleus. The velocity, $V_{\mathrm{LSR}}=229 \pm 3 \mathrm{~km} \mathrm{~s}^{-1}$, is identical to that obtained by Pence, and in the following discussion, we adopt this as the systemic velocity.

\section{THE DISTRIBUTION OF STELLAR AND INTERSTELLAR MASS}

To casual inspection, neither the infrared nor the CO distributions show obvious fine-scale structure correlated with the dark dust lanes or the bright, optical emission regions (except for the specific examples noted above). For the CO data, the general lack of correspondence with dust features may be attributed to inadequate angular resolution; however, this cannot be the case for the 1.27 and $2.2 \mu \mathrm{m}$ scans. For the latter, we believe the explanation lies in the fact that the near-infrared emission is fairly insensitive to extinction and arises mainly from the older disk population giant stars (Aaronson 1977) that are more uniformly distributed than the young, massive stars. (For the standard dust extinction curve in our own Galaxy, the ratio of visual to $2.2 \mu \mathrm{m}$ opacity is approximately $11: 1$ [cf. Becklin et al. 1978].)

In this section the observed kinematics and flux distributions are used to analyze semiquantitatively the distribution of stars and gas in the disk of NGC 253. The galactic mass derived from the rotational velocities is based on the assumption of Keplerian motion in a spherical mass distribution, ignoring both the disk shape and the bar. Derivation of the $\mathrm{H}_{2}$ distribution is based upon comparison with $\mathrm{CO}$ data in our own Galaxy and thus does not take account of possible variation in metallicity or the different properties of clouds in NGC 253.

a) Masses and Column Densities of $\mathrm{H}_{2}$ and $\mathrm{H}_{\mathrm{I}}$

To derive the abundance of $\mathrm{H}_{2}$ from the $\mathrm{CO}$ data we adopt a linear relationship between the $\mathrm{CO}$ line flux and the $\mathrm{H}_{2}$ column density, with a constant of proportionality estimated. from observations of giant molecular clouds in the Galaxy. Based on the observations in our own Galaxy, an empirical relation for face-on column density integrated through the galactic disk is

$$
N_{\mathrm{H}_{2}}=4 \times 10^{20} I_{\mathrm{CO}} \cos i \mathrm{H}_{2} \mathrm{~cm}^{-2} \text {, }
$$

where $I_{\mathrm{CO}}$ is the $\mathrm{CO}$ line integral $\left(\mathrm{K} \mathrm{km} \mathrm{s}^{-1}\right)$, and $i$ is the inclination angle of the galaxy (cf. Young and Scoville 1982). The inclusion of the term cos $i$ makes this a "face-on" column density. The estimates derived for NGC 253 are given in Table 4. The column density of $\mathrm{H}_{2}$ on the nucleus is $2.2 \times 10^{22} \mathrm{~cm}^{-2}$, while that at the secondary peak $(R=3 \mathrm{kpc})$ is a factor of 10 less. Also included in Table 4 are estimates for the $\mathrm{H}$ I column density obtained by $21 \mathrm{~cm}$ aperture-synthesis measurements with a $2^{\prime} \times 3^{\prime}$ beam (Combes, Gottesman, and Weliachew 1977).

In general, the $\mathrm{H}$ I column density exhibits a much flatter 

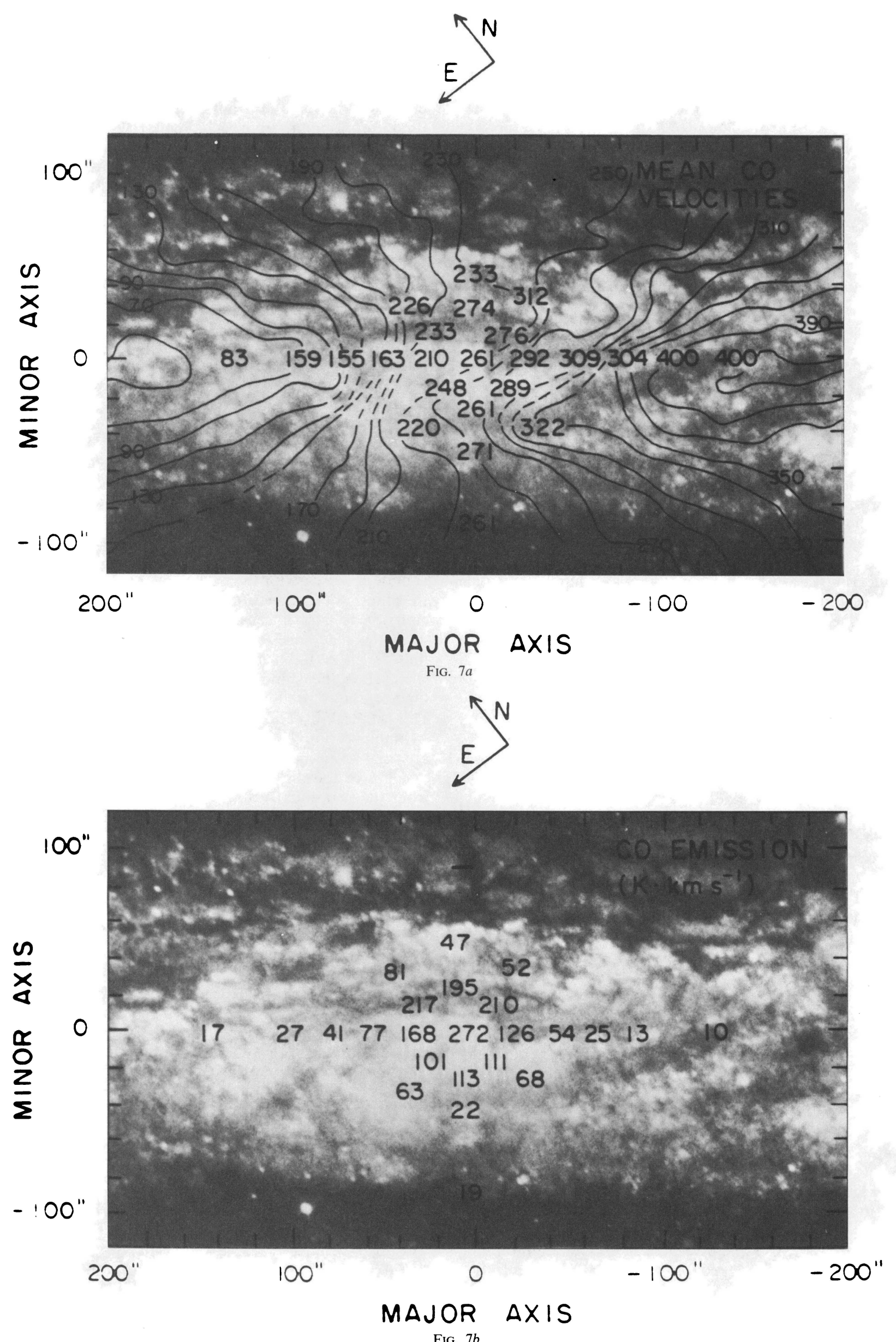

FIG. 7.-The mean CO velocities (Fig. 7a) and integrated intensities (Fig. 7b) are shown for the central region of NGC 253. Note the correspondence of high $I_{\mathrm{CO}}$ on the NW side of the major axis with the high optical extinction.

( ) American Astronomical Society - Provided by the NASA Astrophysics Data System 
TABLE 4

SURFACE DENSITIIES IN NGC 253

\begin{tabular}{|c|c|c|c|c|c|c|}
\hline \multicolumn{2}{|c|}{ RADIUS } & \multirow{2}{*}{$\begin{array}{c}N_{\mathrm{H}_{2}}^{{ }^{\mathrm{a}}} \\
\left(10^{21} \mathrm{~cm}^{-2}\right)\end{array}$} & \multirow{2}{*}{$\begin{array}{c}N_{\mathrm{HI}}^{\mathrm{b}} \\
\left(10^{21} \mathrm{~cm}^{-2}\right)\end{array}$} & \multirow[b]{2}{*}{$2 N_{\mathrm{H}_{2}} / N_{\mathrm{HI}}$} & \multirow{2}{*}{$\begin{array}{c}N_{K}^{\mathrm{c}} \\
\left(\mu \mathrm{Jy} \mathrm{pc}^{-2}\right)\end{array}$} & \multirow{2}{*}{$\begin{array}{c}N_{B}^{\mathrm{d}} \\
\left(L_{\odot} \mathrm{pc}^{-2}\right)\end{array}$} \\
\hline$(")$ & (pc) & & & & & \\
\hline 0 & 0 & 22 & 2.7 & 16 & 4.8 & 159 \\
\hline 11 & 185 & $\ldots$ & $\ldots$ & $\ldots$ & 1.3 & 99 \\
\hline 22 & 370 & 12 & 2.7 & & 0.55 & 76 \\
\hline 45 & 740 & 5.2 & 2.6 & 4 & 0.30 & 69 \\
\hline 68 & 1110 & 2.6 & 2.6 & 2 & 0.22 & 58 \\
\hline 90 & 1480 & 1.6 & 2.5 & 1.3 & 0.17 & 58 \\
\hline 135 & 2220 & 1.1 & 1.6 & 1.4 & 0.15 & 48 \\
\hline 180 & 2960 & 1.6 & 0.7 & 4.6 & 0.15 & 40 \\
\hline 225 & 3700 & 1.4 & 1.1 & 2.5 & 0.080 & 28 \\
\hline 270 & 4440 & $<0.4$ & 1.2 & $<0.7$ & 0.044 & 19 \\
\hline 315 & 5180 & $<0.4$ & 0.9 & $\ldots$ & 0.035 & 17 \\
\hline 500 & 8240 & $\ldots$ & $\ldots$ & $\ldots$ & 0.023 & 16 \\
\hline
\end{tabular}

a Obtained from the CO line fluxes via eq. (1). Since $N_{\mathrm{H}_{2}}$ includes a factor $\cos i$, it is a face-on column density.

${ }^{b}$ Face-on $\mathrm{H}$ I column densities from aperture-synthesis study of Combes, Gottesman, and Weliachew 1977 with $2^{\prime} \times 3^{\prime}$ resolution.

c $N_{K}$ is the face-on surface brightness at $2 \mu \mathrm{m}$ along the major axis.

${ }^{d} N_{B}$, the face-on surface brightness in the blue band, was obtained from the major axis surface photometry of Pence 1980 at $10^{\prime \prime}$ resolution and is expressed in solar luminosity units adopting $M_{B \odot}=5.48 \mathrm{mag}$.

radial profile than the $\mathrm{H}_{2}$ (Fig. 8). From the nucleus out to 5 $\mathrm{kpc}$ radius, the $\mathrm{H}$ I density falls only a factor of 3 , while in the same zone the $\mathrm{H}_{2}$ column density falls more than a factor of 50 (see Table 5). In absolute abundance, $\mathrm{H}_{2}$ exceeds $\mathrm{H}$ I out to a radius of $4 \mathrm{kpc}$. In the nucleus, the density of molecular gas exceeds that of atoms by a factor of 16 ; at a radius of $4 \mathrm{kpc}$ they are equivalent.

\section{b) Luminosity Distributions}

It is of interest to compare the ISM distributions derived above with the distributions of optical and near-infrared luminosity. Included in Table 4 are the mean surface brightnesses observed along the major axis in both the blue $(B)$ and

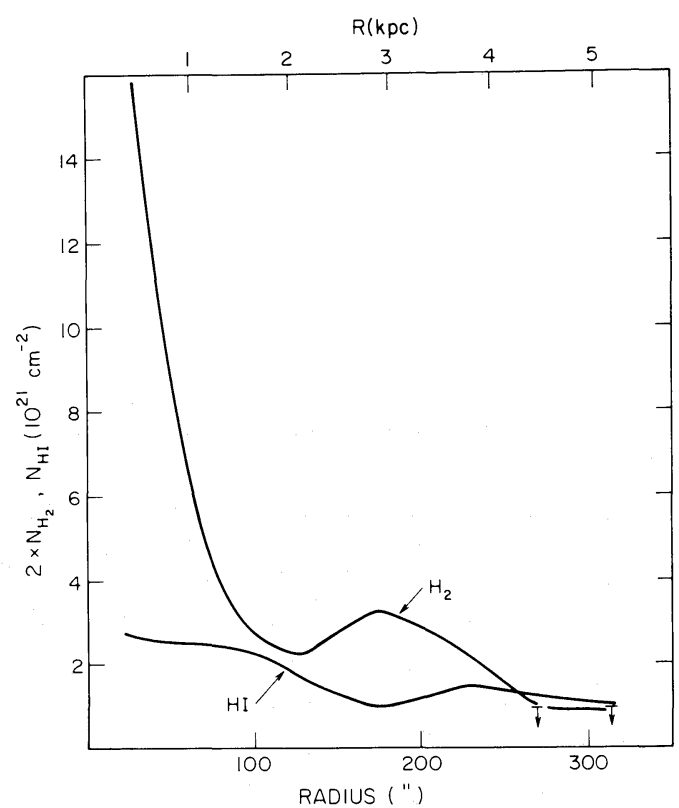

FIG. 8.-The surface densities of $\mathrm{H}_{1}$ and $\mathrm{H}_{2}$ (from Table 4) are shown along the major axis. at $2.2 \mu \mathrm{m}$. The blue surface brightness is expressed in units of luminosity per $\mathrm{pc}^{2}$ and is derived from the $B$ magnitudes based on the solar blue magnitude $M_{B}=5.48$ mag (Allen 1973), with no correction for extinction. This blue luminosity is not an accurate bolometric luminosity. This is especially true in the nucleus, where the far-infrared luminosity is a factor of 3 greater than the blue luminosity derived here. The $2.2 \mu \mathrm{m}$ surface brightness in Table 4 is expressed in units of $\mu \mathrm{Jy} \mathrm{pc}^{-2}$, corrected for the inclination of the galaxy.

\section{c) The Stellar Mass Distribution}

Combining the optical kinematics (Ulrich 1978; Pence 1981) with those of $\mathrm{CO}$, we have estimated the dynamical mass as a function of radius. Outside $100^{\prime \prime}$ radius the velocities are taken from Pence, and in the interior they are taken from Ulrich's data on the northeast side of the major axis (dots in Fig. 1). (As noted in the previous section, the optical velocities on the southwest side are probably affected by foreground obscuration in a dark dust lane). The adopted rotation curve shows a steep ascent to $116 \mathrm{~km} \mathrm{~s}^{-1}$ within only $11^{\prime \prime}$, or $185 \mathrm{pc}$, of the nucleus (Table 5). A local maximum is reached at $45^{\prime \prime}$ followed by a local minimum at $70^{\prime \prime}$. The asymptotic rotational velocity is approximately $200 \mathrm{~km} \mathrm{~s}^{-1}$.

The derived dynamical masses given in Table 5 differ from earlier models principally in being larger at small radius. This difference is due to our conclusion that the optical velocities observed on the west side are too low because of the obscuration.

The estimates for the dynamical mass, $M_{r}$, assume circular orbits about a point mass located in the center of the galaxy. The real distribution of luminous mass is of course a flattened spheroid with considerable mass located outside each radius given in Table 5 . Were the entire mass distribution treated in a self-consistent axisymmetric model, the derived masses at each radius would be somewhat larger. In view of the existence of the bar and the resulting noncircular motions, we could not justify a self-consistent but more involved analysis with an axisymmetric disk. 
TABLE 5

INTEGRATED MASS AND LUMINOSITY DisTRIBUTIONS

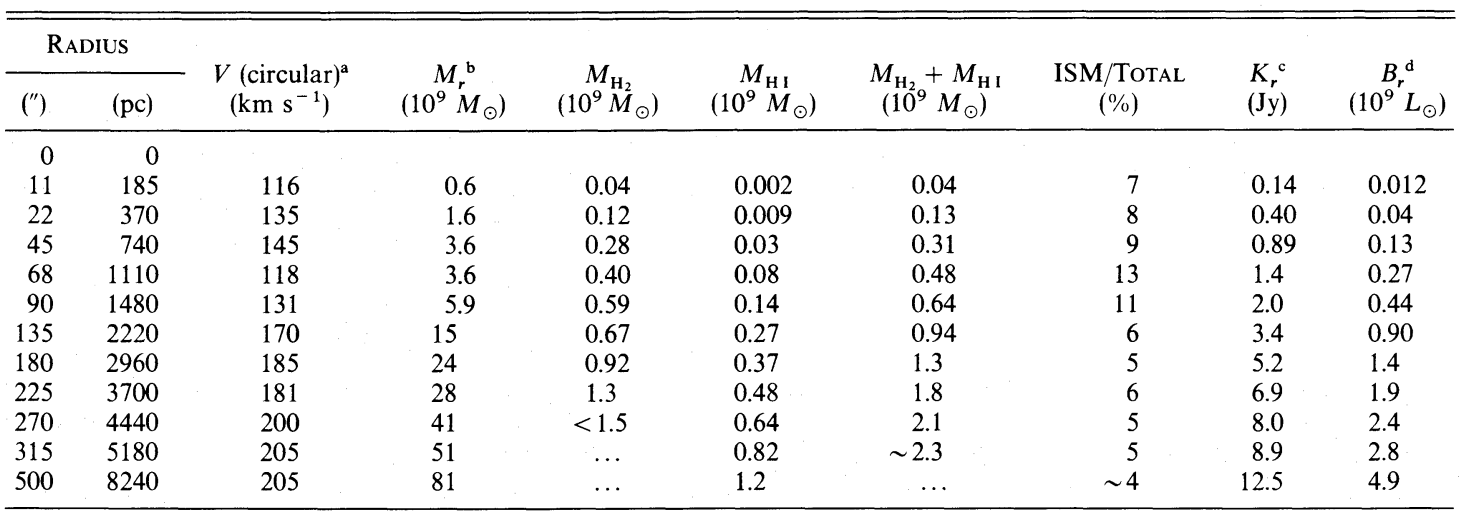

${ }^{a}$ Circular velocities derived from optical emission lines (Pence 1981 at radii greater than $100^{\prime \prime}$ and Ulrich 1978 at smaller radii). Optical velocities on the SW side of the nucleus between $10^{\prime \prime}$ and $90^{\prime \prime}$ are not used on account of likely foreground dust absorption of emission regions in the major axis (see text). Inclination and systemic velocity are given in Table 1.

${ }^{\mathrm{b}}$ Assumes Keplerian orbital motion; then $M / M_{\odot}=2.33 \times 10^{5}\left(V / \mathrm{km} \mathrm{s}^{-1}\right)^{2}(R / \mathrm{kpc})$.

${ }^{\mathrm{c}} K_{\mathrm{r}}$ is the integrated $2 \mu \mathrm{m}$ flux density out to radius $r$ (obtained from $N_{K}$ in Table 4). We assume that the major axis light profile represents the true radial distribution along all azimuthal angles.

${ }_{\mathrm{d}} B_{r}$ is the integrated blue luminosity out to radius $r$ implied by the blue surface density in Table 4. $B_{r}$ is not expected to be an accurate estimate of the total luminosity.

Also included in Table 5 is an estimate of the fraction of the total dynamical mass contained in the interstellar gas $\left(\mathrm{H} \mathrm{I}+\mathrm{H}_{2}\right)$. In the nucleus and most of the disk, the gas fraction is about $5 \%-7 \%$. The total mass of $\mathrm{H}_{2}$ out to $3 \mathrm{kpc}$ radius is $1.1 \times 10^{9} M_{\odot}$, or about $4 \%$ of the dynamical mass in the same area (Table 5). For the central CO beam $(R<400 \mathrm{pc})$, we obtain $M_{\mathrm{H}_{2}}=1.4 \times 10^{8} M_{\odot}$, which is about $9 \%$ of the corresponding dynamical mass. The derived $\mathrm{H}_{2}$ masses at the central beam are, in fact, rather similar to those in the equivalent region of the Milky Way (Sanders, Solomon, and Scoville 1984).

In Figure 9, the dynamical mass, integrated $2 \mu \mathrm{m}$ flux, and integrated $\mathrm{H}_{2}+\mathrm{H}_{\mathrm{I}}$ mass from Table 5 are plotted as a function of radius. The dynamical mass was derived from the rotation curve as described above. The $2.2 \mu \mathrm{m}$ fluxes and the mass of interstellar gas were derived from the surface densities along the major axis (see Table 4) assuming their distributions are circularly symmetric in the disk of the galaxy.

The proportionality seen in Figure 9 between the $2 \mu \mathrm{m}$ luminosity profile and the dynamical mass profile supports the notion that the $2 \mu \mathrm{m}$ flux distribution maps out the structure of the underlying disk mass distribution and is less sensitive to the influence of variable extinction and young stars than the visible light. A possible exception to this is the nuclear source, where there is contamination from hot dust emission at $2 \mu \mathrm{m}$ and from supergiants (cf. Rieke et al. 1980), and where the extinction is substantial ( $A_{2 \mu \mathrm{m}} \sim 0.4 \mathrm{mag}$ ).

\section{DISCUSSION}

The derived mass and luminosity distributions permit a quantitative analysis of the rates of star formation and conversion of interstellar matter into young stars. Using these estimates it is then possible to assess the evolution of the current star formation activity in NGC 253.

\section{a) The Rate of Star Formation}

The total far-infrared luminosity $\left(3 \times 10^{10} L_{\odot}\right)$ measured by Telesco and Harper (1980) for the central $1^{\prime}$ may be translated into a lower limit for the required rate of star formation. Under the assumption that most of this luminosity is produced by $\mathrm{O}$, $\mathrm{B}$, and $\mathrm{A}$ stars, the required rate of consumption of interstellar material into the stars is $2.3 \mathrm{M}_{\odot} \mathrm{yr}^{-1}$ (using eq. [5] of Scoville and Young 1983). Within the same region, the $\mathrm{H}_{2}$ mass was estimated to be $5 \times 10^{8} M_{\odot}$, implying a mean cycling time of $2.5 \times 10^{8} \mathrm{yr}$ for conversion of a typical $\mathrm{H}_{2}$ molecule into stars.

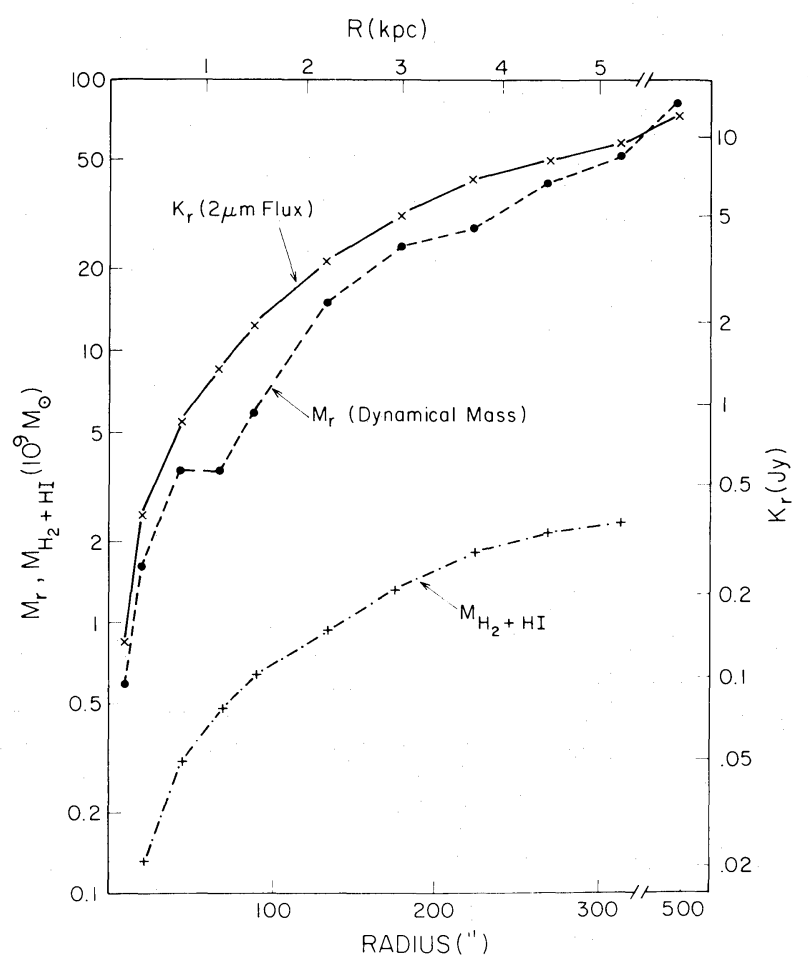

FIG. 9.-The integrated mass distribution $M_{r}$ derived from the rotation curve, the $2 \mu \mathrm{m}$ flux integral (derived from the profile on the major axis), and the integrated ISM mass are shown as a function of radius in NGC 253. 
The estimate of $2.3 \mathrm{M}_{\odot} \mathrm{yr}^{-1}$ for the rate of star formation has no allowance for the formation of lower mass stars or the replenishment of the interstellar medium by mass loss from the high-mass stars. (The degree to which these effects offset each other depends upon the unknown initial mass function.) Nevertheless, it is clear from the above estimate that the ISM in the center of NGC 253 is cycled on a time scale short compared with the age of the galaxy, and unless the gas in the nuclear region is replenished, the current rate of star formation can be sustained no longer than 500 million years.

In their near-infrared spectroscopic and photometric study of M82 and NGC 253, Rieke et al. (1980) found they could model the activity with a burst of star formation but required that the formation of stars of less than $3.5 M_{\odot}$ be strongly inhibited. If this were not the case, then the total mass in the nucleus would quickly build up to the point where it exceeded the limits obtained dynamically. Similar arguments pertain to the analysis presented here. From Table 5 it is seen that the dynamical mass within a radius of $30^{\prime \prime}(R<500 \mathrm{pc})$ is approximately $2 \times 10^{9} M_{\odot}$. At a minimum rate of star formation (including only the massive stars), this dynamical mass would be obtained from the current star formation rate of $2.3 M_{\odot}$ $\mathrm{yr}^{-1}$ within $10^{9} \mathrm{yr}$. For a standard initial mass function (Scalo and Miller 1979), the total mass going into stars later than O, $\mathrm{B}$, and $\mathrm{A}$ will be approximately a factor of 4 greater than that in the $\mathrm{O}, \mathrm{B}$, and $\mathrm{A}$ stars. Thus, for the standard initial mass function, the total dynamical mass would be accumulated in stars within 300 million years. This supports the conclusion of Rieke et al. (1980) that the formation of low-mass stars must be inhibited if the star formation activity lasts of order $10^{8} \mathrm{yr}$, as seems reasonable.

\section{b) The Role of the Bar}

In the previous section is was shown that the current rate of star formation implied by the observed luminosity in the nucleus of NGC 253 cannot be sustained longer than $3 \times 10^{8}$ $\mathrm{yr}$, given the supply of gas in the nucleus. Possibly relevant to this issue are the gasdynamics expected in barred galaxies. The orbits of gas within a bar potential have been modeled theoretically by Roberts, Huntley, and van Albada (1979). They find that the orbits are highly elliptical and, in fact, are not strictly closed. That is, if there exists a high viscosity within the bar due to a high gas density, then the orbits rapidly decay inward. The clear implication of their work is that the gas may flow inward in the bar, fed from the outer disk of the galaxy to the nucleus. Under proper circumstances, a bar may thus replenish the galactic nuclear sources with fresh gas. Observational studies addressing this possibility have been made by Simkin, $\mathrm{Su}$, and Schwartz (1980) and Su and Simkin (1980), who found a high percentage of Seyfert galaxies to have bars in their central regions and a "stepped" light profile like that seen in the near-infrared scans of NGC 253. The detection of a bar in NGC 253 lends further support to the notion that such structures can play a role in building up a supply of fresh gas in the galactic nucleus. It is also interesting that the bar structure detected in the near-infrared mapping extends in the disk out to a radius comparable with that of the secondary maximum seen in the molecular hydrogen. It is thus possible that the bar may aid in the formation of a ring of molecular clouds at about $4 \mathrm{kpc}$ radius.

From the limits to the dynamical mass in this region it is clear that the rate of star formation cannot have persisted much longer than $10^{9} \mathrm{yr}$. This fact rules out the possibility that the current level of activity in the nucleus could have persisted over the age of the galaxy sustained by a continual resupply of fresh gas from the outer disk of the galaxy. An important question therefore remains, why is a burst of star formation observed at this time? If the bar and its associated gas, currently seen in the nucleus, have always existed there, why did the burst not start (and finish) much earlier in the history of the galaxy?

\section{CONCLUSIONS}

The principal results of our near-infrared continuum and millimeter line mapping of NGC 253 are as follows:

1. The near-infrared strip scans show two components: a nuclear source of radius $10^{\prime \prime}-20^{\prime \prime}$ and an inner disk approximately $180^{\prime \prime}$ in radius. A two-dimensional mapping of the inner disk component at $10^{\prime \prime}$ resolution at $\lambda=2.2 \mu \mathrm{m}$ reveals a barlike feature at position angle $17^{\circ}$ east of the major axis in NGC 253.

2. Both the nuclear and the inner disk sources show excess $2.2 \mu \mathrm{m}$ radiation compared with the colors normally seen in disk galaxies, which cannot be entirely explained by heavy extinction. This excess is probably contributed by hot dust associated with star formation regions.

3. The CO radial distribution exhibits a steep falloff from the nucleus out to a local minimum at a radius of $135^{\prime \prime}$. A secondary maximum occurs on both sides of the nucleus along the major axis at $200^{\prime \prime}$, corresponding to the outer radius of the inner disk seen in the near-infrared. Along the minor axis, stronger $\mathrm{CO}$ emission is seen to the northwest than to the southeast. This asymmetry correlates well with the optical obscuration in the northwest.

4. The CO kinematics show much greater symmetry about the center of the galaxy along the major axis than do the optical velocities, especially within the inner $150^{\prime \prime}$ radius. Based on the presence of strong $\mathrm{CO}$ emission to the northwest of the nucleus, it is suggested that the optical velocities measured on the southwest major axis underestimate the true velocity field in the galaxy because of high extinction. A new rotation curve is adopted, which has a steep rise close to the nucleus, indicating a greater dynamical mass within the central $20^{\prime \prime}$ than estimated in earlier analyses of optical data.

5. The radial distribution for $\mathrm{H}_{2}$ obtained from the $\mathrm{CO}$ observations exhibits a stronger falloff with radius than the previously measured $\mathrm{H}$ I distribution. Out to a radius of $4 \mathrm{kpc}$, the surface density of $\mathrm{H}_{2}$ exceeds that of $\mathrm{H}$ I, while outside 4 $\mathrm{kpc}$, the $\mathrm{H}$ I probably dominates. The total mass of molecular clouds within $4 \mathrm{kpc}$ is estimated to be approximately $2 \times 10^{9}$ $M_{\odot}$. An equivalent mass of atomic hydrogen is not attained until $10 \mathrm{kpc}$ radius. The fraction of the total dynamical mass contained in the interstellar gas $\left(\mathrm{H}_{2}+\mathrm{H} \mathrm{I}\right)$ is equal to approximately $7 \%$ in the nucleus and approximately $4 \%$ in the disk of the galaxy.

6. If the luminosity observed in the far-infrared from the nucleus is produced by young massive stars, the implied star formation rate is $2.3 M_{\odot} \mathrm{yr}^{-1}$ in $\mathrm{O}, \mathrm{B}$, and A stars. From the derived mass of molecular material in the nucleus, the mean cycling time must be shorter than $3 \times 10^{8} \mathrm{yr}$. With the present supply of interstellar gas, in the inner disk the current rate of star formation cannot be sustained over a galactic lifetime.

7. We speculate that fresh gas from the inner disk of the galaxy is brought into the nucleat region, which is undergoing a burst of star formation, via the bar structure. Theoretical models for the dynamics of gas in a barlike potential indicate 
that the gas cloud orbits will not be closed. That is, in the event that the viscosity is high, the orbits will decay rapidly, with gas being brought from the extremities of the bar to the central region. We speculate that this is the mechanism by which fresh gas from the inner disk of the galaxy is fed to the nuclear region, which is undergoing a burst of star formation.
It is a pleasure to acknowledge the assistance of Denise Chapman-Taylor and helpful discussions with Jay Elias and Steve Strom. This is contribution \# 570 of the Five College Astronomy Department. The infrared work at Palomar Observatory is supported by the National Science Foundation under grant AST 80-17335.

\section{REFERENCES}

Aaronson, M. 1977, Ph.D. thesis, Harvard University.

Allen, C. W. 1973, Astrophysical Quantities (3d ed.; London: Athlone Press).

Beck, S. C., Lacy, J. H., and Geballe, T. R. 1979, Ap. J., 231, 28.

Becklin, E. E., Fomalont, E., and Neugebauer, G. 1973, Ap. J. (Letters), 181 L27.

Becklin, E. E., Matthews, K., Neugebauer, G., and Willner, S. P. 1978, Ap. J., 223, 831 .

Cohen, J. G., Frogel, J. A., Persson, S. E., and Elias, J. H. 1981, Ap. J., 249, 481

Combes, F., Gottesman, S., and Weliachew, L. 1977, Astr. Ap., 59, 181.

Demoulin, M. H., and Burbidge, E. M. 1970, Ap. J., 159, 799.

Elias, J. H., Frogel, J. A., Mathews, K., and Neugebauer, G. 1982, A.J., 87, 1029.

Fabbiano, G., and Trinchieri, G. 1984, Ap. J., 286, 491

Frogel, J. A., Persson, S. E., Aaronson, M., and Matthews, K. 1978, Ap. J., 220 75.

Johnson, H. L. 1966, Ann. Rev, Astr. Ap. 4, 193

Kleinmann, S. G., Hall, D. N. B., and Scoville, N. Z. 1984, in preparation.

Pence, W. D. 1980, Ap. J., 239, 54. 1981, Ap. J., 247, 473 .
Rickard, L. J., Palmer, P., Morris, M., Turner, B. E., and Zuckerman, B. 1977, Ap. J., 213,673.

Rieke, G. H., Lebofsky, M. J., Thompson, R. I., Low, F. J., and Tokunaga, A. T. 1980, Ap. J., 233, 24

Roberts, W. W., Huntley, J. M., and van Albada, G. D. 1979, Ap. J., 233, 67.

Sandage, A. 1961, The Hubble Atlas of Galaxies (Washington: Carnegie Institution of Washington)

Sanders, D. B., Solomon, P. M., and Scoville, N. Z. 1984, Ap. J., 276, 182.

Scalo, J. M., and Miller, G. E. 1979, Ap. J., 233, 596.

Scoville, N. Z., and Young, J. S. 1983, Ap. J., 265, 148

Simkin, S. M., Su, H. J., and Schwartz, M. P. 1980, Ap. J., 237, 404

Su, H. J., and Simkin, S. M. 1980, Ap. J. (Letters), 238, L1.

Telesco, C. M., and Harper, D. A. 1980, Ap. J., 235, 392.

Turner, J. L., and Ho, P. T. P. 1983, Ap. J. (Letters), 268, L79.

Ulrich, M. H. 1978, Ap. J., 219, 424.

Wynn-Williams, C. G., Becklin, E. E., Matthews, K., and Neugebauer, G. 1979, M.N.R.A.S., 189, 163 .

Young, J. S., and Scoville, N. Z. 1982, Ap. J., 258, 467.

K. Matthews, G. Neugebauer, and B. T. Solfer: California Institute of Technology, 320-47, Pasadena, CA 91125

N. SCoviLle: California Institute of Technology, 105-24, Pasadena, CA 91125

JAYNE YeRKA and J. S. Young: Astronomy Department, University of Massachusetts, Amherst, MA 01003 